\title{
DESIGN AND CHARACTERIZATION OF PERMANENT MAGNETIC SOLENOIDS FOR REGAE
}

\author{
M. Hachmann, K. Floettmann, F. Mayet, DESY, Hamburg, Germany \\ T. Gehrke, DKFZ, Heidelberg, Germany
}

\begin{abstract}
REGAE is a small electron linear accelerator at DESY. In order to focus short and low charged electron bunches down to a few $\mu$ m permanent magnetic solenoids were designed, assembled and field measurements were done.

Due to a shortage of space close to the operation area an in-vacuum solution has been chosen. Furthermore a tworing design made of wedges has been preferred in terms of beam dynamic issues. To keep the field quality of a piecewise built magnet still high a sorting algorithm for the wedge arrangement has been developed and used for the construction of the magnets. The magnetic field of these solenoids has been measured with high precision and has been compared to the simulated magnetic field.
\end{abstract}

\section{INTRODUCTION}

The Relativistic Electron Gun for Atomic Exploration (REGAE) is a small $5 \mathrm{MeV}$ linear accelerator at DESY in Hamburg, which produces short, low emittance electron bunches. It originally was meant for temporal resolving electron diffraction experiments [1]. But two further experiments are currently planned at REGAE. First, an external injection experiment for Laser Wakefield Acceleration (LWA) [2] will be performed in the framework of the LAOLA collaboration (LAboratory fOr Laser- and beam-driven plasma Acceleration). This experiment will provide a method for the reconstruction of the electric field distribution within a linear plasma wakefield. Second one is an extension of the original experiment. A time resolving high energy Transmission Electron Microscope (TEM) will be set up.

Both experiments require strong focusing magnets inside the new target chamber at REGAE. Permanent magnetic solenoids (PMSs) can provide the needed focusing strength due to their enormous surface current density, while having compact dimensions at the same time. Since short and strong solenoids, as required for REGAE, exhibit a distinct nonlinearity, the induced emittance growth is relatively large and has to be minimized as far as possible. Furthermore, the focusing strength is not adjustable and 3D in-vacuum movers are required for positioning the magnets. Due to the chosen movers a weight limitation for the magnets reveals as an additional requirement. Overcoming these difficulties PMSs are an interesting alternative when a low energy beam has to be strongly focused.

\section{DESIGN}

A strong focusing is needed to generate a small transverse beam size for the external injection experiment and for a large magnification in the transmission electron microscope.

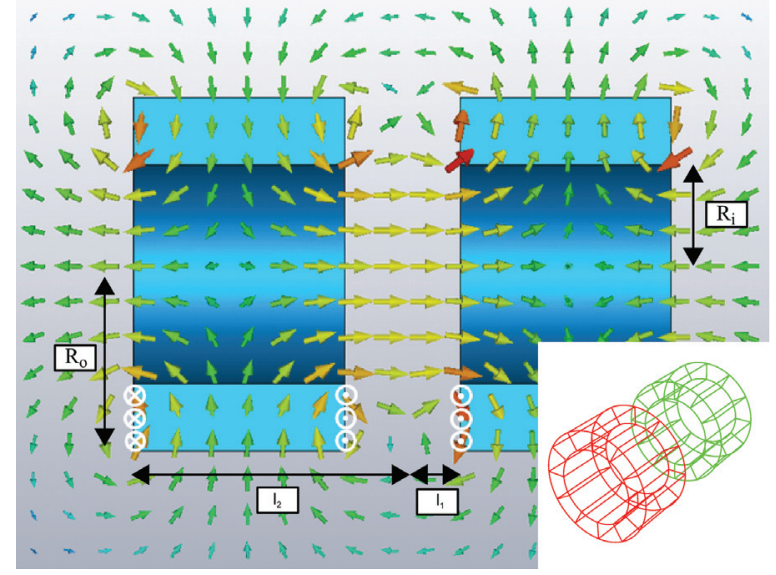

Figure 1: CST simulation of two radially magnetized rings (blue) and the conceptional wedge-based design.

This is achieved by the presented PMS design [3]. A second demand is a small emittance growth induced by the PMS The investigations of different designs have shown that the induced emittance growth for two radially magnetized rings is considerably smaller than for an axially magnetized ring, if a weight limitation has to be applied. In the particular case at REGAE, a reduction of the emittance growth by $65 \%$ is feasible. Mimicking the magnetic field of the single axially magnetized ring with two radially magnetized rings allows for a larger influence on the field shape since a third free parameter, the distance $d=2 l_{1}$ between both rings, is introduced. The PMS dimensions (in $\mathrm{mm}$ ) depicted in Fig. 1 are as follows $R_{o}=25.4, R_{i}=17, l_{1}=7.8$ and $l_{2}=44.8$ while the weight is just $0.628 \mathrm{~kg}$. The focal length for a $5 \mathrm{MeV}$ electron beam is $\sim 0.2 \mathrm{~m}$. For technical reasons radially magnetized rings need to be assembled from wedges. The imperfections of the wedges call for a sorting algorithm in order to preserve the field quality.

\section{FIELD DESCRIPTION AND SORTING ALGORITHM}

\section{Field Model}

Due to the necessity of a model which describes the resulting field of 24 wedges we developed a simple model: each wedge is described by current loops covering the surface (Fig. 2 b)), where each loop can be divided into four straight parts. The magnetic field can be calculated by means of BiotSavart's law for a straight wire. The magnetization $\mathbf{M}$ of a wedge is defined by the direction and its magnitude which were measured by the manufacturer and can be translated into a tilt of the current loops or a variation of the current, 
respectively. The average current is chosen in a way that it reproduces the measured maximum longitudinal magnetic field. The manufacturing errors are added to this average current proportionally.

In order to improve the performance of the calculation routine it is possible to reduce the number of current loops $N$ per wedge but in contrast gain some field uncertainties. In Fig. 2 a) these uncertainties are illustrated as the deviation from the converged $2^{\text {nd }}$ field integral $(N \rightarrow \infty)$, which is proportional to the focus strength of a solenoid

$$
\int_{-\infty}^{\infty} B_{z, 0}{ }^{2}(z) \mathrm{d} z
$$

Here $B_{z, 0}$ is the longitudinal on-axis magnetic field component. We decided to use $N=20$ current loops per wedge and end up with a field integral accuracy of $\sim 1 \%$.

a)

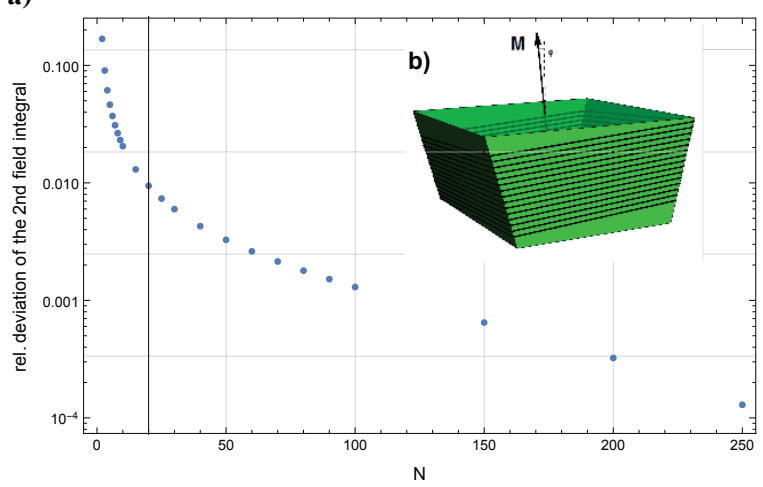

Figure 2: a) Relative deviation of the $2^{\text {nd }}$ field integral for different number of current loops. b) Model of a wedge with current loops and a tilted magnetization.

\section{Sorting Algorithm}

Since the total magnetic field of the two-ring setup is simply given as the superposition of the individual magnetic fields of the wedges (in theory) an optimal configuration of the 24 wedges can be found. The optimal configuration could for example be a case where errors in the direction of the magnetization vector $\mathbf{M}_{i j}$ ( $i$ denotes ring 1 or 2 and $j \in[1,12])$ compensate each other. In order to quantify the definition of the optimal configuration the fit criterion

$$
\sum_{k=0}^{N}\left(I\left(r, \theta_{k}\right)_{\mathrm{p}}-I\left(r, \theta_{k}\right)_{\mathrm{np}}\right)^{2}
$$

with

$$
I\left(r, \theta_{k}\right)=\left.\int_{z_{\min }}^{z_{\max }} B_{r}(r, z) \mathrm{d} z\right|_{\theta_{k}}
$$

was used, where $B_{r}(r, z)$ is the radial magnetic field at distance $r$ from the geometrical z-axis, $\theta_{k}$ the $k$ th rotation angle around the $\mathrm{z}$-axis, $\mathrm{p}$ and $\mathrm{np}$ denote a solenoid with flawless and flawed wedges, respectively. This fitness value has to be minimized in order to achieve maximum field symmetry and to minimize higher order (quadrupole, sextupole, etc.) field components, which might have detrimental effect on beam dynamics.

Using the computationally simple field description shown above an algorithm has been developed with the goal to find the optimal permutation for each ring given the measured magnetization data provided by the manufacturer. This was necessary because the calculation time by a brute force computation would not be feasible $\left(1 \mathrm{ring} \rightarrow 12\right.$ ! $\approx 5 \times 10^{8}$ permutations). The algorithm is a two-step process. The first step consists of a numerical least-square algorithm, which determines a rough starting point for the second part. Here all available wedges - including spare wedges - are taken into account. In each iteration three actions can be performed:

- Swap with pool (incl. spares)

- Swap inside the rings

- Flip around radial axis.

The second step is based on the concept of simulated annealing [4]. Simulated annealing tries to find the global minimum of a fitness function like Eq. 1 by treating the system as a thermodynamical system with falling temperature $T$. For each iteration the fitness function $f(x)$ is determined. Also $T$ is lowered according to a predefined sequence. In our case each iteration consists of swapping wedges inside the rings or flipping them around the radial axis. $x$ corresponds to a certain permutation of both rings whereas $x_{\mathrm{opt}}$ is the current best solution. If $f(x) \leq f\left(x_{\mathrm{opt}}\right), x_{\mathrm{opt}}=x$. If $f(x)>f\left(x_{\mathrm{opt}}\right), x_{\mathrm{opt}}=x$ only with a probability

$$
\exp \left(-\frac{\left.f\left(x_{\mathrm{opt}}\right)-f(x)\right)}{T}\right) .
$$

From Eq. 2 it can be seen that for low temperatures $T$ the probability of choosing the permutation decreases, whereas for high $T$ the algorithm tends to jump out of minima more often. This helps to avoid trapping in local minima.

\section{Results}

The permutations provided by the algorithm were then used to determine emittance growth. To this end full 3D field maps were calculated for both the flawed and flawless wedge case using the analytical field model. These were then used for particle tracking using ASTRA [5]. Because the emittance growth depends on the initial beam parameters the results are only valid and comparable for the chosen parameters as well. We used a $5 \mathrm{MeV}$ beam with an RMS beam size of $600 \mu \mathrm{m}$. Two assemblies were found with a relative emittance growth $\epsilon_{\mathrm{np}} / \epsilon_{\mathrm{p}}$ of 1.04 and 1.15 , respectively. An arbitrary assembly reaches an average relative emittance growth of $\sim 1.30$.

\section{FIELD MEASUREMENT}

\section{D-Hall Probe}

Measuring the magnetic field of a geometrical small magnet with a high precision is challenging. In order to com- 
pare the field simulations with a measurement we decided for the Metrolab Three-axis Hall Magnetometer THM1176HF [6] which provides the required accuracy. With a sensor housing of $5.1 \mathrm{~mm} \times 1.3 \mathrm{~mm}$ the geometrical dimensions of the probe are small enough to measure the magnetic field inside the PMS. Furthermore its small active volume of $(150 \times 150 \times 10) \mu \mathrm{m}^{3}$ is sufficient to measure the absolute field despite the high field gradients.

The Hall probe was calibrated relatively to an NMR Teslameter. The absolute as well as the relative accuracy meet our requirements of $10^{-4}$. From a linear regression of the absolute accuracy measurement follows for the slope 0.999 98(9) and for the offset $1.08(3) \times 10^{-4}$. The number inside the brackets denotes the uncertainty of the last digit.

\section{Magnetic Field Measurement and Post-Processing}

The field measurements were done with a 3D linear stage with a minimal step size of $12.5 \mu \mathrm{m}$. The PMS was fixed on a triple-axis adjustment table. The solenoidal field (Fig. 3) itself offers the possibility to align the linear stage relatively to the magnetic field of the PMS. In its transverse plane at the position of the maximum longitudinal field it has only a longitudinal field component. This fact can be used to align the horizontal and vertical axes of the Hall probe. At the longitudinal position of the zero-crossing of the magnetic field all field components are equal to zero. These two points define the symmetry axis of the solenoid which can be used to align the PMS with respect to the longitudinal axes of the linear stage. Because already small deviations of the alignment can cause big deviations of the measured from the simulated magnetic field a post-processing alignment is necessary to compare simulation and measurement. There are 9 degrees of freedom for the alignment: 3 translational $(x, y, z)$ and 3 rotational degrees $\left(\beta_{x}, \beta_{y}, \beta_{z}\right)$ of the PMS with respect to the linear stage and 3 rotational degrees $\left(\alpha_{x}, \alpha_{y}, \alpha_{z}\right)$ of the Hall probe itself. The indices denote the rotation axes and $(x, y, z)$ the horizontal, vertical and longitudinal axes, respectively. The aforementioned field simulation code has been extended by 3 rotational degrees for the PMS geometry. As described, the other two rotational degrees of the probe are included by the field measurement itself. The calculated values are $\alpha_{x}=-7.41(4) \times 10^{-3} \mathrm{rad}$ and $\alpha_{y}=4.43(4) \times 10^{-3} \mathrm{rad}$. Because the simulated field is a rectangular array the 3 translational degrees are introduced as a shift of the simulated field with respect to the measured one. In order to do this the resolution of the simulated field has to be higher. As a consequence, the translation alignment is discrete and not continuous like the rotational alignment. The rotation around the longitudinal axis of the probe $\alpha_{z}$ is introduced as a simple rotation of the field vector around the $z$-axis.

For the fitting routine a simple least-square criterion is chosen:

$$
\chi^{2}=\sum_{i}^{N} \frac{\left(\mathbf{B}_{\mathbf{m}, \mathbf{i}}-\mathbf{B}_{\mathbf{s}, \mathbf{i}}\right)^{2}}{\sigma_{\mathbf{B}}}
$$

where $\mathbf{B}_{\mathbf{m}}$ is the measured magnetic field vector, $\mathbf{B}_{\mathbf{s}}$ is the simulated magnetic field vector, $\sigma_{\mathbf{B}}$ is the standard deviation of the repeatedly measured field and $N$ is the sample size. In order to proof the goodness of the fit the reduced Chi Square $\widetilde{\chi}^{2}=\chi^{2} /(N-n-1)$ is introduced which should be close to 1 .

For one of the PMS the field was measured and compared to both assembled PMS models as well as the ideal PMS model with flawless wedges. For the fit 27 magnetic field vectors around each aforementioned zero-crossings were taken into account. The step size of the grid was $(50 \times 50 \times 50) \mu \mathrm{m}^{3}$. The results of the fits are shown in Tab. 1 . The resolution of the simulated grid was $(16.7 \times 16.7 \times 12.5) \mu \mathrm{m}^{3}$ and hence the minimal translation step widths of the fit routine.

Table 1: Comparing Fit Results for Both PMS Assemblies as Well as for the Ideal Solenoid Model With Flawless Wedges

\begin{tabular}{lccc}
\hline & PMS 1 & PMS 2 & Ideal \\
\hline $\mathbf{x}[\mathrm{mm}]$ & 0.0333 & -0.0333 & 0.0333 \\
$\mathbf{y}[\mathrm{mm}]$ & -0.0333 & 0 & -0.0167 \\
$\mathbf{z}[\mathrm{mm}]$ & -0.0375 & -0.025 & -0.0375 \\
$\beta_{\mathbf{x}}[\mathrm{rad}]$ & $10^{-4}$ & 0 & $2 \times 10^{-4}$ \\
$\beta_{\mathbf{y}}[\mathrm{rad}]$ & 0.0033 & 0.0032 & 0.0033 \\
$\beta_{\mathbf{z}}[\mathrm{rad}]$ & 0 & $17 \pi / 16$ & 0 \\
$\alpha_{\mathbf{z}}[\mathrm{rad}]$ & 0.024 & 0.024 & 0.031 \\
$\widetilde{\chi}^{2}$ & 0.439 & 0.460 & 0.460 \\
\hline
\end{tabular}

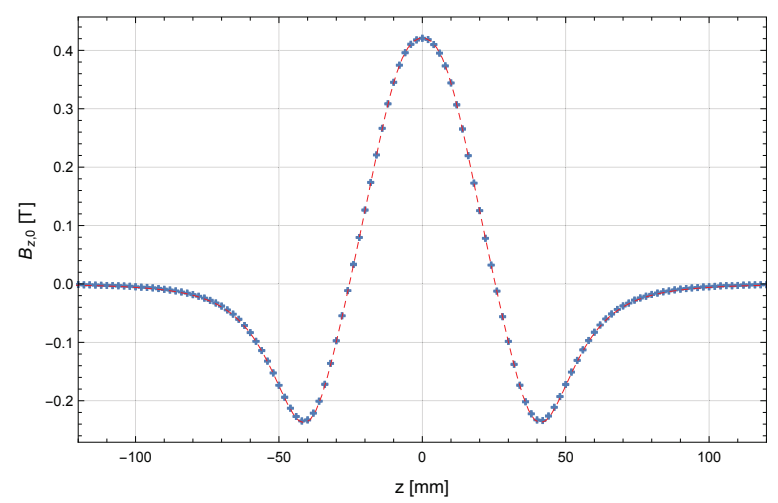

Figure 3: Measured (blue dots) and simulated (red dashed) on-axis magnetic field $B_{z}$.

\section{CONCLUSION}

The goodness of the fit indicates an overestimation of the measurement errors. Furthermore the results for the two different PMS are almost the same which indicates that it is not possible to resolve the smallest deviations of the two PMS. Nevertheless we were able to develop an analytical and fast magnetic field simulation tool which is very general and not limited to solenoidal fields. Furthermore the comparison between the measurement and the simulation was successful despite the challenging magnetic field measurement.

\section{7: Accelerator Technology}




\section{REFERENCES}

[1] J.R. Dwyer et al., "Femtosecond electron diffraction: 'making the molecular movie' ", Philosophical Transactions of the royal society, 364:741-778 (2006).

[2] B. Zeitler et al., "Merging conventional and laser wakefield accelerators", Proceedings SPIE 8779 (2013).

[3] T. Gehrke, "Design of Permanent Magnetic Solenoids for REGAE", Master thesis, Hamburg, Germany (2013).
[4] S. Kirkpatrick et al., "Optimization by simulated annealing", Science (1983), 220 (4598), pp. 671-680.

[5] K. Floettmann, "ASTRA", http://www.desy.de/ $\sim \operatorname{mpyflo}$

[6] Metrolab Technology SA, "Three-axis Magnetometers THM1176 and TFM1186: User's manual”, Version 1.3 (2014), http://thm1176.metrolab.com 